\title{
The effects of memory, extra-memorial functions, and neuro-imaging on the prognosis of operated and non-operated temporal lobe epilepsy patients
}

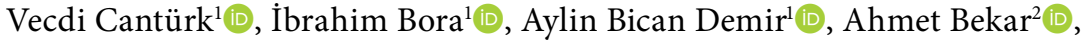 \\ Bahattin Hakyemez ${ }^{3}$, Fevzi Tamgaç ${ }^{4}$, Nevin Türkeş ${ }^{5}$ \\ ${ }^{1}$ Department of Neurology, Uludağ University Faculty of Medicine, Bursa, Turkey \\ ${ }^{2}$ Department of Neurosurgery, Uludağ University Faculty of Medicine, Bursa, Turkey \\ ${ }^{3}$ Department of Radiology, Uludag University Faculty of Medicine, Bursa, Turkey \\ ${ }^{4}$ Department of Nuclear Medicine, Uludağ University Faculty of Medicine, Bursa, Turkey \\ ${ }^{5}$ Department of Neurology and Neuropsychology Laboratory, Uludağ University Faculty of Medicine Bursa, Turkey
}

\begin{abstract}
Objectives: In this study, we aimed to evaluate memory and extra-memorial functions and neuroimaging in operated and non-operated temporal lobe epilepsy (TLE) patients.

Patients and methods: Medical files of a total of 90 patients (47 males, 43 females; median age: 33 years; range, 19 to 68 years) who were diagnosed with TLE at Uludag University, Faculty of Medicine, Department of Neurology between January 2002 and December 2011 were retrospectively and prospectively analyzed. All patients were hospitalized in the video-electroencephalography (EEG) unit of the clinic. Seizure follow-up, video-EEG, cranial magnetic resonance imaging (MRI), and positron emission tomography (PET), and neuropsychometric testing (NPT) were performed in all patients. Thirty-seven of these patients underwent amygdalohippocampectomy + anterior temporal lobectomy by neurosurgery department, while the remaining 53 patients were followed with medical treatment.

Results: Video-EEG, MRI, PET, and NPT showed similar results in terms of lateralization. The operated patients had improved memory and extra-memorial functions in the following controls. A significant decrease in seizure frequency was found in the operated patients after surgery. A significant improvement was observed in the quality of life with reduced seizure frequency and better cognitive functions.

Conclusion: Based on our study results, the diagnostic value of verbal memory tests alone is $63 \%$ on the left, whereas the diagnostic value of the Wechsler Memory Scale visual memory test alone is $69 \%$ on the right.
\end{abstract}

Keywords: Memorial function, neuro-imaging, surgery, temporal lobe epilepsy.

Epilepsy is a condition which appears in the central nervous system due to different causes and is characterized by repetitive and excessive neuronal discharges. The incidence of epilepsy differs among populations; however, the incidence for industrialized countries is ranges from 20 to 70/100,000 per year, while the cumulative incidence for lifetime is about $3 \%$ and active epilepsy prevalence is 4 to $10 / 1,000 .^{[1]}$
Temporal lobe epilepsy (TLE) is usually resistant to antiepileptic drug treatment. Epilepsy surgery provides a complete seizure control in 60 to $80 \%$ of these patients. ${ }^{[2-4]}$ The most successful results in epilepsy surgery are obtained in patients with mesial TLE (MTLE). The achievement rates after treatment due to different surgical methods vary between 33 and 90\% (average 70\%). ${ }^{[2-4]}$ 
The selection of eligible candidates for surgery through a rigorous examination before surgery plays an important role in surgery achievement. Epileptic focus should be detected by interictal electroencephalography (EEG) and long-term video-EEG monitoring; the diagnosis should be confirmed by magnetic resonance imaging (MRI) and magnetic resonance spectroscopy (MRS) among functional imaging methods and positron emission tomography (PET), neuropsychological tests, Wada test and functional MRI (fMRI).

Although memory problems appear during follow-up period of patients with TLE, problems beyond the memory can be also seen. ${ }^{[5]}$ In addition, seizure-free status, as well as problems in memory and out of the memory, affect the quality of life after surgery. Imaging methods performed and efficiency of neuropsychological tests should be searched in this aspect.

Neuropsychology is an assisting method to identify patients who have a risk for disruption in cognitive processes after the surgical procedure. Since $80 \%$ of the epilepsy procedures are performed in the temporal area, assessment of the learning and memory is quite important. ${ }^{[6]}$ Indeed, neuropsychological tests are part of a comprehensive preoperative assessment for most types of epilepsy surgery. They may be useful to identify cognitive risks that may arise from the surgical procedure itself in epilepsy surgery.

In some studies, the risk of postoperative verbal memory disruption increased in left anterior lobectomy patients, compared to patients who were followed without operation, if verbal memory of these patients were normal. ${ }^{[7]}$ In other studies assessing the postoperative verbal memory, postoperative verbal disruption was not affected by the presence of absence of removal of the anterior hippocampus, while this memory disruption was related to preoperative functionality of the removed tissue rather than the volume of it. ${ }^{[8]}$ Performance decrease is observed

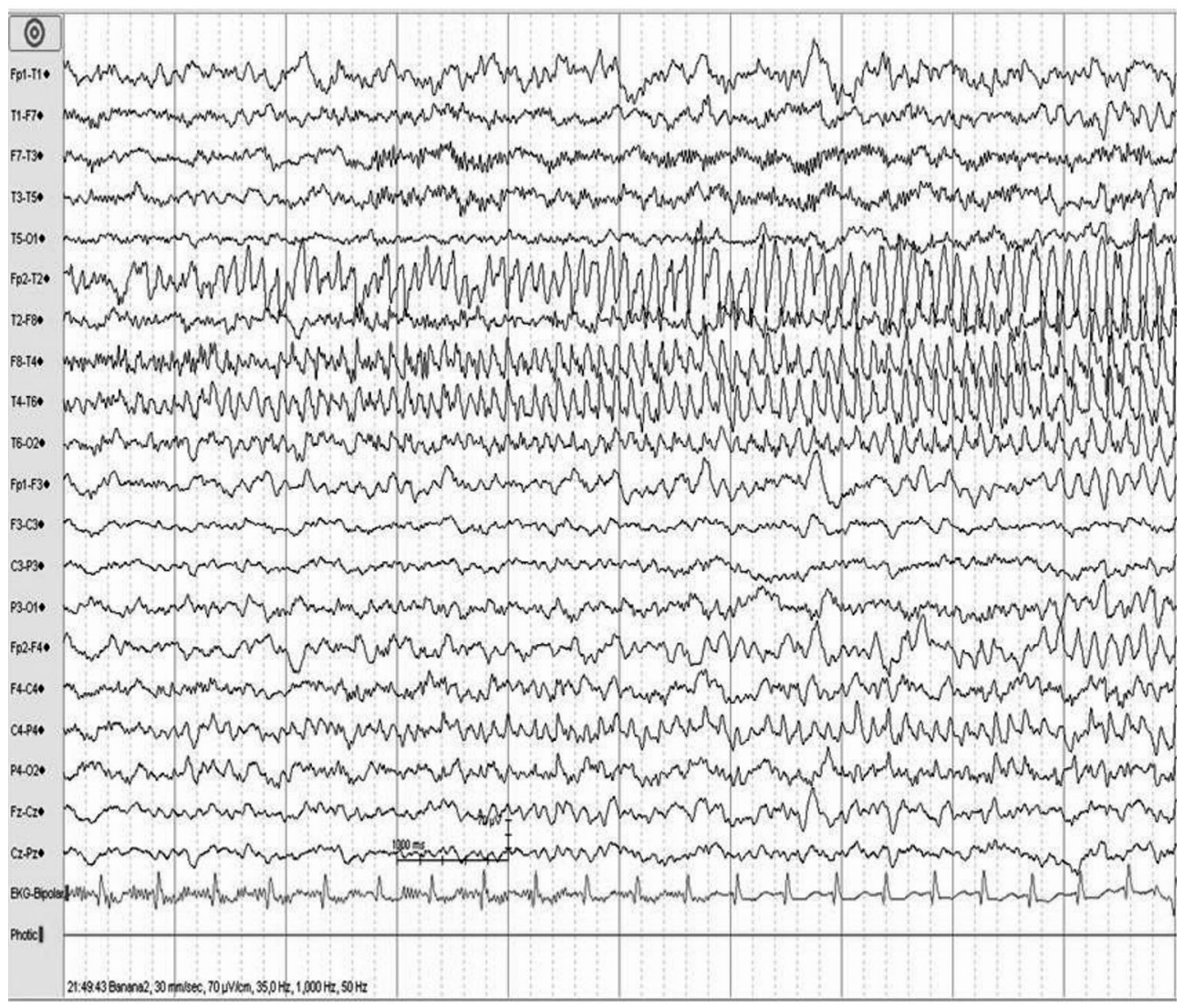

Figure 1. Electroencephalography right temporal rhythmic 7-8 Hz. Sharp wave-like theta activity. 
in neuropsychometric tests, especially in frrontal and visospatial functions in TLE patients. ${ }^{[9]}$ The significance of these findings is still unclear currently. In the present study, we aimed to investigate memory and extra-memorial functions and neuroimaging in operated and non-operated TLE patients.

\section{PATIENTS AND METHODS}

Medical files of a total of 90 patients (47 males, 43 females; median age: 33 years; range, 19 to 68 years) who were diagnosed with TLE at Uludag University, Faculty of Medicine, Department of Neurology between January 2002 and December 2011 were retrospectively and prospectively analyzed. Patients included in this study were hospitalized in video-EEG monitoring unit and diagnosed with TLE after seizure. All patients underwent EEG monitoring (Figure 1), cranial MRI and NPT. The PET scan and MRS (Figure 2) were performed, if necessary. A written informed consent was obtained from each patient. The study protocol was approved by the Uludağ University Clinical Trials Ethics Committee. The study was conducted in accordance with the principles of the Declaration of Helsinki.

Among these patients, amygdalohippocampectomy + anterior temporal lobectomy were performed in 37 patients by the neurosurgery department and 53 patients consisted of those who were considered to benefit from the operation or those who did not accept the operation, even if the operation was indicated and they were followed medically. The biopsy materials of 37 operated patients were examined by the pathologists. The NPT was performed in all patients before the procedure whether surgery was performed and the first and second NPT procedures were performed after three months and one year, respectively. The second control NPT results were compared with the previous procedure. Control NPT was performed in 29 of 53 patients who were not operated and these were compared with the NPT results of the operated patients. The MRS was performed in 67 patients and PET was performed in 40 patients. Neuroimaging results of all patients were compared with each other. The patients operated and followed by medical treatment were compared in terms of neuropsychological test results, neuroimaging data, and seizure frequency.
The patients with TLE who were resistant to the medical treatment and over 18 years were included in the study. Those having progressive characteristics of the disease, mental retardation (IQ $<70)$, and those who withdrew their consent were excluded.

\section{Magnetic resonance imaging protocol}

The MRI of all patients included in the study was performed using the $1.5 \mathrm{~T}$ device (MagnetomVision Plus, Siemens, Erlangen, Germany) by standard head coil according to the epilepsy protocol. Slices were placed as vertical - oblique to the long axis of the hippocampus
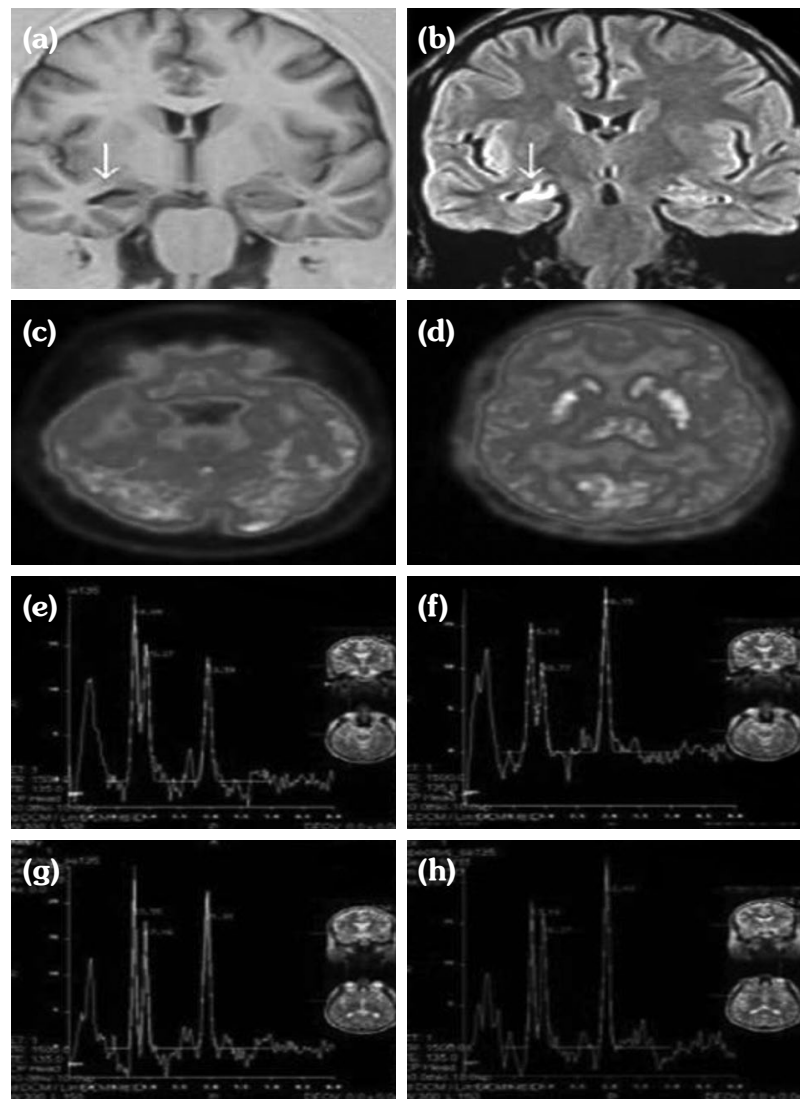

Figure 2. Right MTS: (a) T1 IR sequence in the right hippocampal; decrease in atrophy and in homogeneity. (b) Flair sequence right hippocampal; clear intensity increase. (c, d) PET/BT compared to the left, decreased metabolism in the right temporal lobe and insular cortex. $(\boldsymbol{e}, \mathbf{f})$ Right hippocampal NAA reduction in MRS, significant increase in Cho and Cre is observed. On the left side of a normal MRS. ( $(\mathbf{g}, \mathbf{h})$ Right insular cortex NAA reduction in MRS significant increase in Cho decrease is observed.

MTS: Mesial temporal sclerosis; PET: Positron emission tomography; CT: Computed tomography; NAA: N-acetylaspartate; MRS: Magnetic resonance spectroscopy. 
on the coronal plane. The images were enlarged three times at least and the limits of entorhinal cortex were drawn manually. Entorhinal cortex and hippocampal volumes of all study and control groups were measured by the same researcher.

\section{Magnetic resonance spectroscopy technique}

The MRS was performed in 67 of patients using a circular polarized head bandage in $1.5 \mathrm{~T}$ super magnet.

\section{Analysis}

Lateralization of the epileptic side according to the MRS findings obtained from the mesial temporal lobes was performed based on the data obtained from 32 healthy individuals in the radiology department. Spectroscopic data from mesial temporal lobes was obtained from NAA, Cho and $\mathrm{Cr}$. NAA/Cho+Cr, NAA/Cho, NAA/Cr and $\mathrm{Cho} / \mathrm{Cr}$ ratios, and the mean and standard deviation (SD) values were calculated. In the control cases, 2SD below of mean parameters were accepted as a limit value for hippocampal damage.

\section{Fluorodeoxyglucose (FDG)-positron emission tomography/computed tomography (CT) imaging technique}

The FDG-PET/CT imaging of 40 patients was performed using the Biograph 6 PET/CT scanner (Siemens, Erlangen, Germany) in the Nuclear Medicine Department of our institution and routine FDG-PET/CT imaging protocol was applied to all patients.

\section{Neuropsychological assessment}

In our study, tests which took about $2 \mathrm{~h}$, were applied to all cases by a psychologist (Table 1), and the related brain areas (Table 2) were examined. The battery which was created to analyze neuropsychological test scores of event-related potentials and event-related oscillations during the cognitive activity was called the BILNOT battery. Disruptions in the verbal and visual memory and in other non-memory areas were assessed using the frontal area tests including mental control tests (Wechsler Memory Scale [WMS], mental fluency,

Table 1. Non-memory test

\begin{tabular}{ll}
\hline Tests of verbal memory processes & Verbal memory \\
\hline WMS IV and delayed recall subtests & Visual memory \\
WMS IV the A story of the subtests, B story & Verbal memory and attention sustaining (logical memory) \\
WMS V subtests & Note \\
WMS III & Mental control \\
Fluency tests & Manager functions, perseveration and memory assessment \\
Line test for determining direction & Visuospatial perception and orientation measurement functions \\
Benton Facial Recognition Test & Visuospatial processes \\
Raven Standard Progressive Matrices Test & Regular right thinking, measurement of reasoning abilities \\
Stroop & Response inhibition and change category (information processing speed) \\
\hline WMS: Wechsler Memory Scale. &
\end{tabular}

WMS: Wechsler Memory Scale.

Table 2. Anatomical localization of non-memory tests

\begin{tabular}{|c|c|c|}
\hline Test name & Of the respective brain areas & That measure cognitive feature/process \\
\hline Stroop test & The frontal lobe & $\begin{array}{l}\text { Focused attention, response inhibition upgrade, } \\
\text { disruptive impact resistance, information processing } \\
\text { speed }\end{array}$ \\
\hline Wechsler memory scale & Temporal hippocampus, frontal lobe limbic system & $\begin{array}{l}\text { Attention, concentration, verbal memory, visual memory, } \\
\text { immediate memory, delayed memory }\end{array}$ \\
\hline Number sequence learning & Temporal hippocampus, frontal lobe limbic system & Learning, short-term memory \\
\hline Raven & $\begin{array}{l}\text { The right hemisphere, parietal lobe, common areas } \\
\text { of the brain }\end{array}$ & $\begin{array}{l}\text { Visual spatial perception, to change the category, } \\
\text { working memory, abstraction and correlation, general } \\
\text { skills }\end{array}$ \\
\hline
\end{tabular}


Stroop Test, Raven, shortened Wechsler Adult Intelligence Scale-Revised [WAIS] subtests judging, abstract thinking, arithmetic ability, planning, and Boston Naming Test-) and parietal area tests (visuospatial abilities, face recognition test, planning, and attention tests) were assessed under following four categories and those with disruption over 1 was recorded according to the groups: ${ }^{[10-17]} 1=$ normal, $2=$ mild disruption, $3=$ intermediate disruption, and $4=$ severe disruption.

\section{Statistical analysis}

Statistical analysis was performed using the SPSS version 13.0 software (SPSS Inc., Chicago, IL, USA). Continuous variables were expressed in median (min-max) values, while categorical variables were expressed in number and percentage. The confrontation of continuous variables with a normal distribution was examined using the Shapiro-Wilk test. The Mann-Whitney $U$ test was used to compare continuous data between two independent groups, while the Kruskal-Wallis test was used for multiple comparisons. Meaning of continuous variables comparison of values according to dependent time groups Wilcoxon rank total test was used. The Pearson chisquare and Fisher's chi-square tests were used for comparison of categorical variables between the groups. The Cohen's kappa coefficient was calculated to determine the compliance between the diagnostic tests. A correlation analysis was performed to examine the relationship between variables in continuous and score values and the Pearson and Spearman correlation coefficients were calculated. A $p$ value of $<0.05$ was considered statistically significant.

\section{RESULTS}

Left hand dominancy was seen only in three (3.3\%) patients, whereas right hand dominancy was seen in 87 (96.7\%) patients. There was no significant difference in the dominant hand between the groups $(p=0.266)$. Of the patients operated, 54.1\% were females and $45.9 \%$ were males. Of the patients who were not operated, $43.4 \%$ were females and $56.6 \%$ were males. There was no significant difference in the sex between the operated and non-operated groups $(\mathrm{p}=0.319)$.

Furthermore, there was no statistically significant difference in the education status between the groups $(p=0.757)$. The median age at the time of operation was 32 (range, 16 to 58) years. The median time from the diagnosis to surgery was 17 (range, 2 to 43) months. Overall, the median number of seizures within one year, the median seizure duration for lifetime, the median duration of drug use for lifetime, and the median number of drugs used for lifetime were detected as 77 (range, 6 to 360) one year 19 (range, 4 to 53) lifetime, 24 (range, 2 to 66) lifetime, and four (range, 1 to 7) lifetime, respectively. There was no significant difference in the number of seizures was found between the operated and non-operated groups with a higher number of seizures in the operated patients $(p=0.011)$.

In comparison of NPT scores and controls, the NPT scores differed according to the control 1-NPTA and control 2-NPTA scores, indicating that the NPT results were improved in the controls $(\mathrm{p}<0.05)$ (Table 3). The NPT scores differed according to the control 1 and control 2

Table 3. In comparison of NPT scores and controls, it was detected that NPT scores differ according to Control 1-NPTA and Control 2-NPTA scores

\begin{tabular}{l|lccc}
\hline & & NPTA $(\mathrm{n}=90)$ & C1-NPTA $(\mathrm{n}=66)$ & C2-NPTA $(\mathrm{n}=19)$ \\
\hline \multirow{3}{*}{ Operated patient $(\mathrm{n}=37)$} & Median & 3 & 2 & 2 \\
& Minimum & 1 & 1 & 1 \\
& Maximum & 4 & 4 & 3 \\
Non operated patient $(\mathrm{n}=53)$ & Median & 2.5 & 2 & 2.5 \\
& Minimum & 1 & 1 & 2 \\
& Maximum & 4 & 4 & 3 \\
\hline
\end{tabular}

NPT: Neuropsychometric testing; NPTA: Neuropsychometric test abnormalities (verbal and visual memory tests); C1-NPTA: $1^{\text {st }}$ Control neuropsychometric test abnormalities; C2-NPTA: $2^{\text {nd }}$ Control neuropsychometric test abnormalities. 
Table 4. Comparison of results of EEG, MRI, PET, and NPT

\begin{tabular}{|c|c|c|c|c|}
\hline & & \multicolumn{3}{|c|}{ EEG localization $(n=90)$} \\
\hline & & NPTA $(n=90)$ & C1-NPTA $(n=66)$ & C2-NPTA $(n=19)$ \\
\hline \multirow{3}{*}{ Operated patient $(\mathrm{n}=37)$} & Right focus ( $\mathrm{n}=42$ ) & 32 & 2 & 8 \\
\hline & Left focus $(n=30)$ & 2 & 26 & 2 \\
\hline & Bilateral focus $(n=18)$ & 6 & 9 & 3 \\
\hline \multirow{3}{*}{ MRS-localization $(n=67)$} & Right focus ( $\mathrm{n}=32$ ) & 23 & 2 & 7 \\
\hline & Left focus $(n=25)$ & 0 & 22 & 3 \\
\hline & Bilateral focus $(n=10)$ & 7 & 3 & 0 \\
\hline \multirow{3}{*}{ PET-localization $(n=40)$} & Right focus ( $n=13$ ) & 9 & 2 & 2 \\
\hline & Left focus $(n=14)$ & 1 & 11 & 2 \\
\hline & Bilateral focus $(n=13)$ & 9 & 3 & 1 \\
\hline \multirow{3}{*}{ NPT-lateralization $(\mathrm{n}=90)$} & Right focus ( $\mathrm{n}=29$ ) & 18 & 5 & 6 \\
\hline & Left focus $(n=32)$ & 6 & 23 & 3 \\
\hline & Bilateral focus $(\mathrm{n}=29)$ & 16 & 9 & 4 \\
\hline
\end{tabular}

EEG: Electroencephalography; MRI: Magnetic resonance imaging; PET: Positron emission tomography; NPT: Neuropsychometric testing; NPTA: Neuropsychometric test abnormalities (verbal and visual memory tests); C1-NPTA: $1^{\text {st }}$ Control neuropsychometric test abnormalities; C2-NPTA: $2^{\text {nd }}$ Control neuropsychometric test abnormalities; MRS: Magnetic resonance spectroscopy.

scores for the patients who underwent surgery, indicating that the NPT results were improved in the operated patients $(p<0.05)$. However, no significant difference was found between the NPT scores and control 1 scores in non-operated patients, indicating that the NPT results remained unchanged in the non-operated patients ( $p>0.05)$.

In terms of EEG lateralization according to MRI, MRS, PET, and NPT-lateralization, it was compatible in terms of the focus detection side $(p<0.05)$ (Table 4). In terms of MRI lateralization according to the MRS, PET, and NPT-lateralization, it was compatible in terms of focus detection side $(\mathrm{p}<0.05)$ (Table 5). In terms of PET lateralization according to NPT-lateralization, it was compatible in terms of the focus detection side $(p<0.05)$ (Table 6).

No disruption was detected in non-memory tests of 37 patients, whereas function disruption was detected in the frontal tests only in 40 patients and, in three patients, in parietal tests only and in both frontal and parietal tests in 10 patients. The PET scan of $91.9 \%$ of the patients whose non-memory test performance was reported as normal; hypometabolism was detected in the frontal area and in other areas in $2.7 \%$ in $5.4 \%$

Table 5. Magnetic resonance imaging lateralization according to MRS, PET, and NPT-lateralization

\begin{tabular}{|c|c|c|c|c|}
\hline & & \multicolumn{3}{|c|}{ MRI-lateralization $(n=90)$} \\
\hline & & $\begin{array}{l}\text { Right focus } \\
(\mathrm{n}=42)\end{array}$ & $\begin{array}{l}\text { Frontal } \\
(\mathrm{n}=30)\end{array}$ & $\begin{array}{l}\text { Bilateral focus } \\
\qquad(\mathrm{n}=18)\end{array}$ \\
\hline \multirow{3}{*}{ MRS-lateralization $(n=67)$} & Right temporal $(\mathrm{n}=32)$ & 30 & 0 & 2 \\
\hline & Left temporal $(\mathrm{n}=25)$ & 0 & 21 & 4 \\
\hline & Bilateral focus $(\mathrm{n}=10)$ & 5 & 2 & 3 \\
\hline \multirow{3}{*}{ PET-lateralization $(\mathrm{n}=40)$} & Right temporal $(n=13)$ & 10 & 2 & 1 \\
\hline & Left temporal $(\mathrm{n}=14)$ & 2 & 11 & 1 \\
\hline & Bilateral focus ( $\mathrm{n}=13$ ) & 9 & 1 & 3 \\
\hline \multirow{3}{*}{ NPT-lateralization $(\mathrm{n}=90)$} & Right temporal $(\mathrm{n}=29)$ & 22 & 3 & 4 \\
\hline & Left temporal $(\mathrm{n}=32)$ & 6 & 18 & 8 \\
\hline & Bilateral focus ( $\mathrm{n}=29$ ) & 14 & 9 & 6 \\
\hline
\end{tabular}

MRS: Magnetic resonance spectroscopy; PET: Positron emission tomography; NPT: Neuropsychometric testing; MRI: Magnetic resonance imaging. 
Table 6. Positron emission tomography lateralization according to NPT-lateralization

\begin{tabular}{llccc}
\hline \multicolumn{2}{l}{} & \multicolumn{3}{c}{ PET-lateralization $(\mathrm{n}=40)$} \\
\cline { 2 - 5 } & $\begin{array}{c}\text { Right focus } \\
(\mathrm{n}=13)\end{array}$ & $\begin{array}{c}\text { Frontal } \\
(\mathrm{n}=14)\end{array}$ & $\begin{array}{c}\text { Bilateral focus } \\
(\mathrm{n}=13)\end{array}$ \\
\hline \multirow{3}{*}{ NPT-lateralization $(\mathrm{n}=90)$} & Right temporal $(\mathrm{n}=29)$ & 7 & 3 & 3 \\
& Left temporal $(\mathrm{n}=32)$ & 2 & 7 & 3 \\
& Bilateral focus $(\mathrm{n}=29)$ & 4 & 4 & 7 \\
\hline
\end{tabular}

NPT: Neuropsychometric testing; PET: Positron emission tomography.

Table 7. Comparison of the first and control non-memory tests in the study population

\begin{tabular}{lllccc}
\hline & & \multicolumn{4}{c}{ C1-NPT-CNM (n=66) } \\
\cline { 3 - 6 } & & $\begin{array}{c}\text { Normal } \\
(\mathrm{n}=38)\end{array}$ & $\begin{array}{c}\text { Frontal } \\
(\mathrm{n}=21)\end{array}$ & $\begin{array}{c}\text { Parietal } \\
(\mathrm{n}=2)\end{array}$ & $\begin{array}{c}\text { Parietal + frontal } \\
(\mathrm{n}=5)\end{array}$ \\
\hline \multirow{3}{*}{ NPT-CNM (n=66) } & Normal $(\mathrm{n}=12)$ & 23 & 6 & 0 & 1 \\
& Frontal $(\mathrm{n}=19)$ & 12 & 15 & 0 & 0 \\
& Parietal $(\mathrm{n}=1)$ & 0 & 0 & 1 & 0 \\
& Parietal + frontal $(\mathrm{n}=5)$ & 3 & 0 & 1 & 4 \\
\hline
\end{tabular}

NPT: Neuropsychometric testing; C-1-NPT-CNM: Control-1 Changes in non-memory tests of neuropsychometrics; NPT-CNM: Changes in non-memory tests of neuropsychometrics.

of the patients, respectively. Hypometabolism on the frontal area was detected by PET in only $10 \%$ of the patients with impaired frontal functions in non-memory tests, whereas PET result was normal in $88 \%$ patients. There was no area compatible with hypometabolism on PET scans of the patients with non-memory problems in NPT (frontal Area tests (Mental Control tests (WMS), Mental Fluency Test, Stroop Test, Raven, Shortened WAIS subtests; judging, abstract thinking, arithmetic ability, planning, Boston Naming Test) and parietal area tests (visuospatial abilities, face recognition test, planning, and attention tests), except for the temporal lobe ( $p>0.01)$.A correlation was observed between the NPT-CNM and control 1 NPT-CNM and there was no improvement in performances of the patients in non-memory tests, when the first NPT and first control NPT results were compared ( $p>0.05)$ (Table 7). In addition, there was no correlation between the NPT-CNM and control 1 NPT-CNM in the operated patients and there was an improvement in performances of the patients in non-memory tests, when the first NPT and first control NPT were compared $(p<0.05)$ (Table 8). There was a compliance between the NPT-CNM and control-1- NPT-CNM in patients who were not operated, and there was no improvement in performances of these patients in the non-memory tests, when the first NPT and first control NPT were compared ( $p>0.05)$ (Table 9).

Table 8. Comparison of the first and control non-memory tests in operated patients

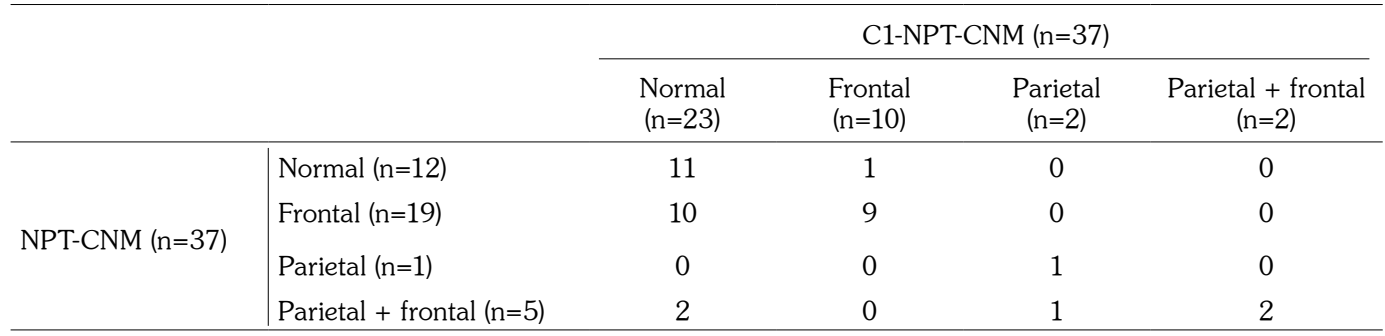

NPT: Neuropsychometric testing; C-1-NPT-CNM: Control-1 Changes in non-memory tests of neuropsychometrics; NPT-CNM: Changes in non-memory tests of neuropsychometrics. 
Table 9. Comparison of the first and control non-memory tests in non-operated patients

\begin{tabular}{l|lcccc}
\hline & & \multicolumn{4}{c}{ C1-NPT-CNM $(\mathrm{n}=29)$} \\
\cline { 3 - 6 } & & $\begin{array}{c}\text { Normal } \\
(\mathrm{n}=15)\end{array}$ & $\begin{array}{c}\text { Frontal } \\
(\mathrm{n}=11)\end{array}$ & $\begin{array}{c}\text { Parietal } \\
(\mathrm{n}=0)\end{array}$ & $\begin{array}{c}\text { Parietal + frontal } \\
(\mathrm{n}=3)\end{array}$ \\
\hline \multirow{3}{*}{ NPT-CNM $(\mathrm{n}=29)$} & 12 & 5 & 0 & 1 \\
& Normal $(\mathrm{n}=18)$ & 2 & 6 & 0 & 0 \\
& Frontal $(\mathrm{n}=8)$ & 0 & 0 & 0 & 0 \\
\cline { 2 - 5 } & Parietal $(\mathrm{n}=0)$ & 1 & 0 & 0 & 2 \\
\hline
\end{tabular}

NPT: Neuropsychometric testing; C-1-NPT-CNM: Control-1 Changes in non-memory tests of neuropsychometrics; NPT-CNM: Changes in non-memory tests of neuropsychometrics.

According to the non-memory test results, only WMS Mental Control Test showed a statistically significant compliance in assessment of each test for all frontal functions separately $(p<0.05)$. When non-memory tests were assessed, only visuospatial abilities and facial recognition test showed a statistically significant compliance in assessment of each test for all parietal functions separately $(p<0.05)$.

\section{DISCUSSION}

Mesial temporal sclerosis (MTS) is the most common etiological cause for temporal zonelocated partial seizures which are resistant to the treatment. It is observed in 60 to $80 \%$ of the MRIs taken according to the epilepsy protocol in patients with epilepsy. After surgical treatment, seizure-free life and increase in quality of life was obtained in 33 to $90 \%$ (average $70 \%$ ) patients in whom the hippocampal pathology was found based on clinical signs, EEG monitoring, and NPT and conventional MRI results. ${ }^{[2-4]}$ Therefore, it is of utmost importance to determine the epileptic area and invasion efficiently and accurately before the surgical treatment. The NPTs are used to assist and support the determination of lateralization. Besides, they provide valuable information to compare cognitive functions of the patients before and after surgery. ${ }^{[3]}$

The time spent until an epilepsy surgery varies between 15 and 20 years worldwide. ${ }^{[1,3]}$ The median time was calculated as 17 years in our study. Therefore, to diagnose the resistant epilepsy as soon as possible and to send the patients to surgery during early period are critical steps for the prognosis.
In a study conducted by Hammen et al., ${ }^{[18]}$ MRS and EEG were congruent with each other in determining the affected area. In another study carried out by Meyer et al., ${ }^{[19]}$ the compliance of EEG to determine the lateralization by neuroimages of 43 patients and compliance rates were determined as $77 \%, 58 \%$, and $56 \%$ for PET/EEG, MRI/EEG, and MRS/EEG, respectively. When we examined the cases with right and left temporal pathology in terms of MRI and EEG compliance, we found the MRI-EEG compliance rate on the right temporal area as 76.2\% (32/42) and MRI-EEG compliance rate on the left side as $86.7 \%(26 / 30)$. When we examined the cases with right and left temporal pathology in terms of MRS and EEG compliance, we detected MRS-EEG compliance rate on the right temporal area as $71.9 \%(23 / 32)$ and MRS-EEG compliance rate on the left side as $88 \%(22 / 25)$. We examined the cases with right and left temporal pathology in terms of PET and EEG compliance, we determined the PET-EEG compliance rate on the right temporal area as $69.2 \%(9 / 13)$ and PET-EEG compliance rate on the left side as $78.6 \%(11 / 14)$.

Furthermore, in a study by Kilpatrick et al., ${ }^{[20]}$ operation side of the patients was compared with imaging methods and NPTs and compliance of the operation side with MRI, ictal EEG, PET and NPT were detected as 98\%, 92\%, 93\%, and 51\%, respectively. When the operation results were assessed for MRI and location, the diagnostic value of MRI alone was $91 \%$ on the left and $74 \%$ on the right; in the assessment of operation results for ictal EEG and location, diagnostic value of ictal EEG alone was found to be $82 \%$ on the left and $81 \%$ on the right. When the operation results were assessed in terms of MRS and location, the 
individual diagnostic value of MRS was found as $100 \%$ on the left and $91 \%$ on the right. In the assessment of operation results in terms of PET and location, the diagnostic value of PET individually was detected as $50 \%$ on the left and $70 \%$ on the right and when the operation results were assessed in terms of SBT and location, the diagnostic value of SBT individually was detected as $63 \%$ on the left, whereas the diagnostic value of WMS visual memory test individually was detected as $69 \%$ on the right.

In our study, the rates in PET were detected lower on the left side than those reported in the literature. This can be attributed to the small sample size in our study who had left-sided disease as evidenced by PET $(n=6)$ and visual comparison of both sides in PET assessments. In many studies, sensitivity of PET in TLE varied between 62 and 85\%. ${ }^{[21,22]}$ However, the true reason was that PET scan cannot be applied to every patient, due to its expensive cost in Turkey and that it can be applied for certain indications only.

Review of the literature revealed that, for a total of 129 patients in whom epilepsy surgery was considered, neuropsychological test results complied with the clinical signs and other findings by $40 \%$, resulting in wrong lateralization in $13 \%$ and it presented findings without lateralization in $47 \% .{ }^{[23]}$ Furthermore, the same tests were considered as reliable in determination of lateralization in some studies, whereas they were detected non-reliable in other studies. ${ }^{[6]}$ In general, verbal memory tests are more sensitive than visual memory tests. ${ }^{[23]}$ In addition, a higher verbal memory performance before left anterior temporal lobectomy causes more postoperative memory disruption; however, the risk in the disruption of verbal memory postoperatively is very low in patients who have verbal memory disruption before surgery and have left hippocampal volume loss. ${ }^{[24]}$

When the operation results were assessed in terms of verbal memory tests and location in our study, the diagnostic value of SBT alone was detected $63 \%$ on the left, whereas the diagnostic value of WMS visual memory test alone was detected $69 \%$ on the right based on the WMS visual memory test and location. The sensitivity of these tests was found to be close to those reported in the literature with respect to determine the focus. However, we observed that sensitivity of the verbal memory tests in the literature was higher than our results. These rates mentioned above were obtained from the operated patients. The discrepancy between our study results and the literature findings can be explained by small sample size of left-side operated patients (11/37).

When the first tests of the non-operated patients were compared with the control NPTs, there was no statistically significant change, while there was a statistically significant improvement in the first and second control tests of the operated patients, compared to first test results $(p<0.05)$. Our findings were evaluated with different literature results and, as shown in the previous studies, we observed that surgical treatment had positive effects on the cognitive functions. We believe that post-surgery improvement may be related to amygdalohippocampectomy + anterior temporal lobectomy technique. This indicates that surgical methods applied for epilepsy surgery should be considered carefully, since they may affect the assessment and preservation of memory functions after surgery.

In a study including 23 patients, duration of the seizure was negatively correlated with impaired verbal and visual memory in both left and right TLE patients. ${ }^{[25]}$ Similarly, all patients included in our study were assessed and performances in both verbal and visual memory tests decreased in both operated and nonoperated patients in case of prolonged seizure duration and increased seizure number. We also found that lower first basic performances were related to a lower improvement possibility, while better performances in the first tests were related to an increased improvement possibility.

Low performance was observed in frontal functions and visuospatial functions along with the MTS side associated memory tests in the NPT procedure in some MTS patients. ${ }^{[26,27]}$ The clinical relevance of these findings is still unclear. Nevertheless, we believe that it can be associated with frontal distribution of electrical discharges. One of the objectives of our study was to investigate whether a hypometabolism existed in associated brain areas in PET scans of the patients with such a test performance. In a study including 13 TLE patients, verbal memory levels increased and material specific memory disorder was more common, particularly in patients in whom relative 
asymmetrical hypometabolism was detected on the left hemisphere and thalamus through the PET scan. Furthermore, in a study conducted in Germany in a patient series including 96 TLE cases, an asymmetrically reduced glucose metabolism disorder was detected in the prefrontal area in patients with left dominant speech hemisphere and it was particularly observed in patients who had secondary generalized seizure. ${ }^{[28]}$

In the current study, there was no disorder in non-memory tests of 37 patients, whereas function disorder was detected in only frontal tests in 40 patients, in only parietal tests in three patients, and in both frontal and parietal tests in 10 patients of 90 patients. Hypometabolism on the frontal area was detected by PET in only 10\% of the patients in whom performance reduction was detected in the frontal area functions via nonmemory tests, whereas no pathological activity was detected on the extratemporal areas in $88 \%$. Hypometabolism was not detected on the areas related to PET in none of the patients having reduced performance in the parietal area functions. Consequently, there was no statistically significant area complying with hypometabolism, except for the temporal area similar to their PET scans of the patients who had non-memory performance reduction in NPT $(p>0.05)$.

In another study, verbal fluency, Stroop, and shortened of WAIS subtests were compared for the control and TLE patient groups and disruption was detected in the patients with TLE, particularly for the Stroop and tracing tests. ${ }^{[29]}$ Furthermore, in another study, executive functions and operation memory were compared between the control group and patients with TLE. ${ }^{[26]}$ In this study, the shortened WAIS subtests, visuospatial ability tests, and mental control tests were assessed and disruption was detected, particularly in the shortened WAIS subtests and mental control tests in patients with TLE. Previous studies also showed that visuospatial ability tests disrupted in the patients with right TLE in particular. ${ }^{[27,29,30]}$ In some studies, disruptions were detected in verbal fluency tests and attention tests for patients with TLE. ${ }^{[31,32]}$ In a study by Martin et al., ${ }^{[33]}$ disruption in the Wisconsin Card Sorting (WCS) test was detected in patients with TLE. In the study of Kim et al., ${ }^{[34]}$ WCS test showed a disruption in executive functions in 56\% of the patients with TLE. Furthermore, a better improvement after surgery could be achieved, if the disruption was severe before surgery.

In our study, the Stroop, Raven, shortened WAIS subtests, mental control tests, mental fluency, and attention tests assessing frontal functions similar to the WCS test were used along with the facial recognition test, line direction determination test, and visuospatial abilities among parietal tests. All tests used for frontal area functions were evaluated together in our study and, consequently, it was observed whether any frontal function disorder was present. Then, competences of each test was evaluated to detect frontal function disorder independent from each other. Furthermore, visuospatial ability tests, facial recognition test, and planning tests were assessed together for the parietal area functions and it was examined whether any parietal function disorder was present. Then, abilities of each test to detect parietal function disorder independent from each other were assessed. In our study, the sensitivity of the following tests to detect frontal function disorder were as follows: $56 \%$ for WMS mental control tests, 36\% for mental fluency test, $84 \%$ for attention tests, 30\% for Stroop test, 32\% for Raven, 34\% for WAIS subtests, 38\% for visuospatial ability tests, $8 \%$ for line direction determination test, and 46\% for facial recognition test. In particular, the WMS control tests from frontal function tests may show the frontal disorders significantly $(p<0.05)$. In our study, the sensitivity of the attention tests was higher to show the frontal disorder. However, this was not statistically significantly compatible, since temporal and parietal areas were affected from the functions ( $p>0.05)$. Facial recognition test and visuospatial ability tests included in parietal function tests may significantly show the parietal disorder $(p<0.05)$. Consequently, tests which were used in the literature and corresponded to our tests including WMS mental control tests, attention tests, facial recognition test, and visuospatial ability tests may reflect brain functions accurately in consistent with the literature.

When frontal and parietal area functions were assessed in our study, a significant improvement was detected in test performances measuring these functions after surgery in operated patients $(p<0.05)$. Among the patients who had performance reduction in the frontal 
and parietal area tests in the first NPT, $52.6 \%$ showed normal performances in control NPT. When the first NPT and control NPTs were compared in the patients who were not operated, no change was detected in terms of performance in the frontal and parietal area tests. Also, it was observed that when seizure duration increased, disruption in these functions increased, whereas the seizure type and seizure frequency remained unchanged. This finding indicates that these functions beyond the memory are affected from seizure duration in consistent with the literature. This finding gives rise to thought of that earlier surgical treatment may have positive effects on these functions.

In a study, the seizure remission rate was detected as $72 \%$ for one-year follow-up, whereas $87 \%$ of the patients were in remission for two years of follow-up and such ratio decreased to $74 \%$ for five-year follow-up and to $67 \%$ for 10-year follow-up. ${ }^{[35]}$ Also, the remission rate for one year after surgery was found to be $82 \%$ in another study. ${ }^{[28]}$ In addition, a study including 227 patients showed that earlier surgery was associated with high remission rates after surgery and eventually postoperative quality of life. ${ }^{[36]}$ In this study, remission rate for one year was detected as $81 \%$ and remission rate for five years was $75 \%$. In our study, the patients operated were followed for postoperative seizures for a mean of five years (range, 6 months to 7 years). A significant decrease was detected in the number of seizures in 37 patients after surgery. Four patients had one seizure each and these patients were assessed in $1 \mathrm{C}$ according to the Engel classification. Furthermore, one patient defined an aura complaint. The remission rate for one year was $89 \%$ after the operation. Among the patients who had seizures, three of them had seizure within the first three months, whereas one patient had seizure at the end of first year. Furthermore, among the patients who were followed for a long term, one patient had seizure in the second year and one patient had seizure in the fourth year. As a result, a significant decrease was observed in the number of seizures after the surgical treatment, consistent with the literature.

At the end of the study, the following conclusions were reached:
- Video-EEG, MRI, MRS, PET, and NPT, which assist the diagnosis in patients treatment-resistant TLE, were found to be compatible with each other in terms of lateralization.

- When all diagnostic methods were assessed, accurate diagnosis and lateralization with a high rate were obtained.

- An improvement trend was observed during postoperative follow-up of the patients who had epilepsy surgery in terms of memory and non-memory functions, compared to patients followed with medical treatment.

- In case of mild cognitive disruption, the benefits which could be achieved with surgery increased. Furthermore, memory tests and non-memory tests could provide information about the prognosis of cognitive functions.

- Verbal memory tests, visual memory tests, and WMS mental control tests, attention tests, facial recognition test and visuospatial ability tests included in nonmemory performance tests were found to be sensitive to detect the disorder in associated brain areas. Although non-memory tests and imaging tests did not have high compliance rates for location in the patients who had epilepsy surgery, they provided valuable information for prognosis.

In conclusion, it is obvious that patients who suffer from seizures for long years, have seizure frequently, do not get benefit from multiple drug treatment for a long period, and are exposed to side effects of these drugs can achieve an improved quality of life with early surgical treatment with less remission and enhanced cognitive functions.

\section{Declaration of conflicting interests}

The authors declared no conflicts of interest with respect to the authorship and/or publication of this article.

\section{Funding}

The authors received no financial support for the research and/or authorship of this article. 


\section{REFERENCES}

1. Berkovic SF, Scheffer IE. Genetics of the epilepsies. Curr Opin Neurol 1999;12:177-82.

2. Maehara T, Ohno K. Preoperative factors associated with antiepileptic drug withdrawal following surgery for intractable temporal lobe epilepsy. Neurol Med Chir (Tokyo) 2011;51:344-8.

3. Thom M, Mathern GW, Cross JH, Bertram EH. Mesial temporal lobe epilepsy: How do we improve surgical outcome? Ann Neurol 2010;68:424-34.

4. Choi H, Carlino R, Heiman G, Hauser WA, Gilliam FG. Evaluation of duration of epilepsy prior to temporal lobe epilepsy surgery during the past two decades. Epilepsy Res 2009;86:224-7.

5. Delazer M, Gasperi A, Bartha L, Trinka E, Benke T. Number processing in temporal lobe epilepsy. J Neurol Neurosurg Psychiatry 2004;75:901-3.

6. Kneebone AC. Presurgical neuropsychological evaluation for localization and lateralization of the epileptogenic zone. In: Lüders H, Comair YG, editors. Epilepsy surgery. 2nd ed. Philadelphia: Lippincott Williams \& Wilkins; 2001. p. 487-96.

7. Loring DW. Chelune GJ. Neuropsychological evaluation in epilepsy surgery. In: Lüders $\mathrm{H}$, Comair YG. editors. Epilepsy surgery. 2nd ed. Philadelphia: Lippincott Williams \& Wilkins; 2001. p. 521-4.

8. Loring DW, Lee GP, Meador KJ, Smith JR, Martin $\mathrm{RC}$, Ackell $\mathrm{AB}$, et al. Hippocampal contribution to verbal recent memory following dominant-hemisphere temporal lobectomy. J Clin Exp Neuropsychol 1991;13:575-86.

9. Rausch R, Henry TR, Ary CM, Engel J Jr, Mazziotta $\mathrm{J}$. Asymmetric interictal glucose hypometabolism and cognitive performance in epileptic patients. Arch Neurol 1994;51:139-44.

10. Lezak MD. Neuropsychological Assessment. 3rd ed. Philadelphia: Oxford University Press; 1995.

11. KarakaşS. Bilişsel fonksiyonlarındeğerlendirilmesinde nöropsikolojik testler. Türk Nöroloji Dergisi 2002;8:61-9.

12. McDowell BD, Bayless JD, Moser DJ, Meyers JE, Paulsen JS. Concordance between the CVLT and the WMS-III word lists test. Arch Clin Neuropsychol 2003:19:319-24.

13. Öktem Ö. Sözel bellek süreçleri testi (SBST) - Bir ön çalışma. Nöropsikiyatri Arşivi 1992;29:196-206.

14. Lacritz LH, Barnard HD, Van Ness P, Agostini M, Diaz-Arrastia R, Cullum CM. Qualitative analysis of WMS-III Logical Memory and Visual Reproduction in temporal lobe epilepsy. J Clin Exp Neuropsychol 2004;26:521-30.

15. Kaplan EF. Goodglass H, Weintraub S. The Boston Naming Test. 2nd ed. Philadelphia: Lea \& Febiger; 1983.

16. Öktem Ö. Klinik Nöropsikoloji bakış açısıyla. In: Karakaş S, editör. Bilnot Bataryası El Kitabı. İstanbul: Güneş Tip Kitabevi; 2004. s. 115-32.
17. Zeki S. The visual image in mind and brain. Scientific Amer 1992;26:69-76.

18. Hammen T, Schwarz M, Doelken M, Kerling F, Engelhorn T, Stadlbauer A, et al. 1H-MR spectroscopy indicates severity markers in temporal lobe epilepsy: correlations between metabolic alterations, seizures, and epileptic discharges in EEG. Epilepsia 2007;48:263-9.

19. Meyer PT, Cortés-Blanco A, Pourdehnad M, LevyReis I, Desiderio L, Jang $S$, et al. Inter-modality comparisons of seizure focus lateralization in complex partial seizures. Eur J Nucl Med 2001;28:1529-40.

20. Kilpatrick C, O'Brien T, Matkovic Z, Cook M, Kaye A. Preoperative evaluation for temporal lobe surgery. $\mathrm{J}$ Clin Neurosci 2003;10:535-9.

21. Kim YK, Lee DS, Lee SK, Kim SK, Chung CK, Chang $\mathrm{KH}$, et al. Differential features of metabolic abnormalities between medial and lateral temporal lobe epilepsy: quantitative analysis of (18)F-FDG PET using SPM. J Nucl Med 2003;44:1006-12.

22. Chassoux F, Semah F, Bouilleret V, Landre E, Devaux $\mathrm{B}$, Turak B, et al. Metabolic changes and electroclinical patterns in mesio-temporal lobe epilepsy: a correlative study. Brain 2004;127:164-74.

23. Dodril CB. Overview: Presurgical Neuropsychological Evaluation. In: Lüders H, Comair YG, editors. Epilepsy Surgery. 2nd ed. Philadelphia: Lippincott Williams \& Wilkins; 2001. p. 475-80.

24. Chelune GJ, Najm IM. Risk factors associated with postsurgical decrements in memory. In: Lüders $\mathrm{H}$, Comair YG, editors. Epilepsy surgery. 2nd ed. Philadelphia: Lippincott Williams \& Wilkins; 2001. p. 497-504.

25. Cheung MC, Chan AS, Chan YL, Lam JM, Lam W. Effects of illness duration on memory processing of patients with temporal lobe epilepsy. Epilepsia 2006;47:1320-8.

26. Tudesco Ide S, Vaz LJ, Mantoan MA, Belzunces E, Noffs MH, Caboclo LO, et al. Assessment of working memory in patients with mesial temporal lobe epilepsy associated with unilateral hippocampal sclerosis. Epilepsy Behav 2010;18:223-8.

27. Baddeley A. Working memory: looking back and looking forward. Nat Rev Neurosci 2003;4:829-39.

28. Jokeit H, Seitz RJ, Markowitsch HJ, Neumann N, Witte OW, Ebner A. Prefrontal asymmetric interictal glucose hypometabolism and cognitive impairment in patients with temporal lobe epilepsy. Brain 1997;120:2283-94.

29. Tuchscherer V, Seidenberg M, Pulsipher D, Lancaster M, Guidotti L, Hermann B. Extrahippocampal integrity in temporal lobe epilepsy and cognition: thalamus and executive functioning. Epilepsy Behav 2010;17:478-82.

30. Wagner DD, Sziklas V, Garver KE, Jones-Gotman M. Material-specific lateralization of working memory in the medial temporal lobe. Neuropsychologia 2009;47:112-22. 
31. Keller SS, Baker G, Downes JJ, Roberts N. Quantitative MRI of the prefrontal cortex and executive function in patients with temporal lobe epilepsy. Epilepsy Behav 2009;15:186-95.

32. Gleissner U, Elger CE. The hippocampal contribution to verbal fluency in patients with temporal lobe epilepsy. Cortex 2001;37:55-63.

33. Martin RC, Sawrie SM, Gilliam FG, Palmer CA, Faught E, Morawetz RB, et al. Wisconsin Card Sorting performance in patients with temporal lobe epilepsy: clinical and neuroanatomical correlates. Epilepsia 2000;41:1626-32.
34. Kim $\mathrm{CH}$, Lee SA, Yoo HJ, Kang JK, Lee JK. Executive performance on the Wisconsin Card Sorting Test in mesial temporal lobe epilepsy. Eur Neurol 2007;57:39-46.

35. Kelemen A, Barsi P, Eross L, Vajda J, Czirják S, Borbély $\mathrm{C}$, et al. Long-term outcome after temporal lobe surgery--prediction of late worsening of seizure control. Seizure 2006;15:49-55.

36. Shimizu H, Kawai K, Sunaga S, Sugano H, Yamada T. Hippocampal transection for treatment of left temporal lobe epilepsy with preservation of verbal memory. J Clin Neurosci 2006;13:322-8. 